\title{
Distribución actual y futura del bosque subalpino de Pinus hartwegii Lindl en el Eje Neovolcánico Transversal
}

\section{Current and future distribution of the Pinus hartwegii Lindl subalpine forest in the Tranverse Neovolcanic Belt}

\author{
Ulises Manzanilla-Quiñones', Óscar Alberto Aguirre-Calderón’*, Javier Jiménez-Pérez', Eduardo Javier Treviño-Garza' \\ y José Israel Yerena-Yamallel' \\ 1 Universidad Autónoma de Nuevo León. Facultad de * Autor de correspondencia. \\ Ciencias Forestales. Linares, Nuevo León, México. ｏscar.aguirrecl@uanl.edu.mx
}

\section{RESUMEN}

De acuerdo con las proyecciones del clima a futuro, muchas especies verían reducidas sus áreas de distribución, principalmente, aquellas que habitan en zonas templadas y frías. El objetivo principal del presente fue modelar la distribución actual y bajo escenarios de cambio climático del bosque subalpino de Pinus hartwegii Lindl para 2041-2060 en el Eje Neovolcánico Transversal. Se obtuvieron los registros de Pinus hartwegii Lindl de la plataforma GBIF y campo. Se descargaron las 19 variables bioclimáticas WorldClim para el presente y futuro de los modelos de circulación global CRNMCM5 y HADGEM2_ES con dos forzamientos radiactivos de 4.5 (trayectorias de $\mathrm{CO}_{2}$ constantes) y 8.5 (trayectorias de $\mathrm{CO}_{2}$ altas). Se eliminó la autocorrelación espacial de las variables. Se cargaron las variables y los registros de presencia al programa MaxEnt, utilizando $75 \%$ de los datos para entrenar los modelos y $25 \%$ para validarlos. Se transfirieron los parámetros del mejor modelo para generar los modelos a futuro. Los modelos presentaron un excelente ajuste estadístico (pruebas de entrenamiento y validación > 0.9) para ambos periodos. Las variables determinantes de ambos periodos analizados fueron temperatura promedio anual (BIO1) y altitud (DEM). Los escenarios de cambio climático predicen un aumento en temperatura promedio anual entre $0.7^{\circ} \mathrm{C}$ y $2.1^{\circ} \mathrm{C}$ y reducciones entre $11.7 \%$ y $26.9 \%$ en el nicho subalpino de Pinus hartwegii Lindl., siendo el Nevado de Toluca y el Pico de Orizaba los sitios más susceptibles al cambio climático. Por otra parte, el parque Izta-Popo presentaría un aumento de nicho de 37.4\%.

PALABRAS CLAVE: áreas de distribución, aumento en temperatura promedio anual, bosques de alta montaña, escenarios de cambio climático, nicho ecológico.

\section{ABSTRACT}

According to future climate projections, many species will see their ranges reduced, mainly those that live in cold forests. The aim of the present study was to model the current distribution and climate change scenarios of the Pinus hartwegii Lindl subalpine forest for 20412060 in the Transverse Neovolcanic Belt. Pinus hartwegii Lindl records were obtained from the Global Biodiversity Information Facility (GBIF) and field. The 19 WorldClim bioclimatic variables for the present and future were downloaded from the CRNMCM5 and HADGEM2_ES Global Circulation Models with two radioactive forcings of 4.5 ( $\mathrm{CO}_{2}$ constant pathways) and 8.5 (CO 2 high pathways). The spatial autocorrelation of the variables was eliminated. The environmental variables and presence records were uploaded to the MaxEnt program, where $75 \%$ of the data was used to train the models and $25 \%$ to validate them. The parameters of the best model were transferred to generate future models. The models presented an excellent statistical fit (training and validation tests $>0.9$ ) for both periods. The determining variables of both periods analyzed were mean annual temperature (BIO1) and altitude (DEM). Climate change scenarios predict an increase in average annual temperature between $0.7{ }^{\circ} \mathrm{C}$ and $2.1^{\circ} \mathrm{C}$ and reductions between $11.7 \%$ and $26.9 \%$ in the subalpine niche of Pinus hartwegii Lindl. Nevado de Toluca and Pico de Orizaba are the most susceptible sites to climate change. On the other hand, Izta-Popo Park would present a 37.4\% niche increase.

KEYWORDS: areas of distribution, increase in average anual temperature, high mountain forests, climate change scenarios, ecological niche. 


\section{INTRODUCCIÓN}

El cambio climático es un fenómeno global que se ha ido documentado a partir de la segunda mitad del siglo XX, desde entonces, diversas instituciones como el Panel Intergubernamental de Expertos Sobre Cambio Climático [PCC] (2014) han invertido muchos recursos en el entendimiento del fenómeno y sus consecuencias en la distribución de las especies de flora y fauna (MartínezMeyer, 2005). Hoy en día, la relación que existe entre la biodiversidad y el clima se ha vuelto motivo de preocupación para la comunidad científica de todo el mundo, debido a los efectos y consecuencias del cambio climático sobre la vegetación (Moore y Allard, 2009; IPCC, 2014). El aumento en temperatura y los cambios en precipitación afectan de diversas maneras a las especies, estos cambios modificarían la distribución y diversidad de especies a futuro (Burgmer, Hillerbrand y Pfenninger, 2007; Sáenz-Romero, Rehfeldt, Ortega-Rodríguez, Marín-Togo y Madrigal-Sánchez, 2015).Una de las principales consecuencias directas del cambio climático es la modificación y alteración de la dinámica y funcionamiento de los ecosistemas, en especial, los ecosistemas forestales (Norby, Morgan y Körner, 2007; Moore y Allard, 2009; IPCC, 2014; Islas-Báez, Pérez-Mirada, GonzálezHernández, Romero-Sánchez y Velasco-Bautista, 2015).

La evidencia científica prueba de que las especies siempre han estado expuestas a lo largo de su historia de vida evolutiva a los cambios en el clima, pero en la actualidad, el ritmo de esos cambios supera por mucho a las fluctuaciones del pasado (Thuiller, Lavergne, Roquet, Boulangeat y Araujo, 2011). Ante tales modificaciones en el clima, las especies contemporáneas tendrían tres posibles alternativas: tolerar las alteraciones climáticas, desaparecer o cambiar sus áreas de distribución actual (Sáenz-Romero et al., 2010, 2015; Cruz-Cárdenas et al., 2016).Estudios realizados en diversas latitudes y altitudes señalan a los ecosistemas de bosques de clima boreal y templado como los más sensibles al cambio climático (IPCC, 2007; IPCC 2014).
De acuerdo con los escenarios de cambio climático para México en el siglo XXI, se predice un aumento en temperatura promedio anual de $0.5^{\circ} \mathrm{C}$ a $2{ }^{\circ} \mathrm{C}$ para 2030 , de $2 .{ }^{\circ} \mathrm{C}$ para 2060 y hasta $3.7^{\circ}$ para 2090 (Sáenz-Romero et al., 2010; Comisión Nacional de Áreas Naturales Protegidas [Conanp], 2015).Ante tales escenarios, los bosques secos en México tenderían a expandir sus áreas de distribución (7.4\%); sitios como la reserva de la biosfera Selva el Ocote en Chiapas se volverían más cálidos y secos $\left(0.83{ }^{\circ} \mathrm{C}\right.$ de aumento en temperatura promedio anual) (Manzanilla y Aguirre, 2017), mientras que la superficie de bosque templado vería reducida entre $60 \%$ y $70 \%$ su área de distribución y el aumento en temperatura haría a estos ecosistemas más vulnerables a los incendios forestales (Villers y Trejo, 1998; Ibarra y Huerta, 2016).

Los modelos de distribución de especies (MDE) son herramientas utilizadas para predecir la distribución potencial (actual, histórica y futura) de las especies (Sandoval y Real, 2005; Peterson, 2011; García-Aranda, Méndez-González y Hernández-Arizmendi, 2018; Manzanilla-Quiñones, Martínez-Adriano y AguirreCalderón, 2019). Por otro lado, los modelos de circulación global (MCG) se usan para simular el comportamiento del clima a futuro (Delgado y Suárez, 2009; FernándezEguiarte, Zavala-Hidalgo, Romero-Centeno, CondeÁlvarez y Trejo-Vázquez, 2015), por lo que son una alternativa para evaluar el posible impacto del cambio climático en la distribución de especies (Jeschke y Strayer, 2008; Peterson et al., 2011; García-Aranda et al., 2018). A continuación, se enlistan algunos de los trabajos efectuados sobre modelamiento espacial bajo escenarios de cambio climático para especies de la familia Pinaceae en México:

Sáenz-Romero, Rehfeldt, Duval y Linding-Cisneros, (2012) mencionan que la distribución potencial de Abies religiosa (Kunth) Schtldl y Cham en la reserva de la biosfera Mariposa Monarca podría desaparecer a finales de este siglo. Monterroso-Rivas, Gómez-Díaz y Tinoco-Rueda, (2012) prevén una reducción significativa en las condiciones climáticas aptas en la distribución de seis 
especies de bosque mesófilo de montaña en el estado de Hidalgo para 2050. Gutiérrez y Trejo (2014) predicen una reducción considerable en las condiciones climáticas idóneas en la distribución de cinco especies arbóreas (Pinus ayacahuite, Pinus hartwegii, Abies hickelii, Quercus laurina y Quercus ocoteifolia) del bosque templado en México, los autores prevén que debido a los cambios en el clima se pudieran presentar algunas extinciones locales para 2050. Estrada-Contreras, Equihua, Castillo-Campos y RojasSoto, (2015) señalan que debido al cambio climático, los bosques de coníferas y el bosque mesófilo de montaña del estado de Veracruz reducirían su distribución potencial apta en 16\% y 49\%, respectivamente, para 2050. Sáenz-Romero et al. (2015) predicen disminuciones en la distribución potencial apta de Pinus leiophylla Shiede ex Schtldl y Cham de $35 \%, 50 \%$ y $76 \%$ para 2030, 2060 y 2090 , respectivamente. Islas-Báez et al. (2015) estimaron una reducción de $49.1 \%$ en las condiciones ambientales idóneas del bosque de Abies religiosa (Kunth) Schtldl y Cham en la reserva de la biosfera Mariposa Monarca para 2050. CruzCárdenas et al. (2016) estimaron que la superficie ambiental apta de 12 especies de la familia Pinaceae en el estado de Michoacán se vería reducida $16 \%$ y $40 \%$ para los periodos 2015-2039 y 2075-2099, respectivamente.

Pinus hartwegii Lindl, llamado también el pino de las alturas, se distribuye en México y Guatemala (Farjon, Pérez de la Rosa y Styles, 1997). El taxón crece y forma bosques puros a partir de $3000 \mathrm{~m}$ snm y hasta $3700 \mathrm{~m}$ snm y se le encuentra en forma semiarbustiva a partir de $4000 \mathrm{~m} \mathrm{snm}$ y hasta $4300 \mathrm{~m}$ snm (Farjon et al., 1997). Dentro de ese gradiente altitudinal, en el Eje Neovolcánico Transversal se localizan montañas representativas de ecosistemas subalpinos y alpinos o también llamados páramos de altura en México (Miranda y Hernández, 1963), los cuales son ecosistemas con poca distribución en el país por las condiciones tan específicas en las que se encuentran. Dentro de ese tipo de ecosistema, Pinus hartwegii Lindl resulta ser una especie apropiada para la evaluación del posible impacto del cambio climático a futuro en los bosques de alta montaña o bosques subalpinos puros de la especie en el Eje Neovolcánico Transversal, los cuales han sido poco explorados y estudiados.

\section{OBJETIVOS}

El objetivo principal del presente estudio fue modelar la distribución actual y futura de Pinus hartwegii Lindl en el Eje Neovolcánico Transversal. Se plantearon los siguientes objetivos particulares; 1) modelar la distribución actual y futura (2041-2060) del bosque subalpino de Pinus hartwegii Lindl, 2) determinar las variables ambientales importantes en los periodos actual y futuro, 3) estimar la superficie que se vería reducida por el cambio climático y 4) identificar las áreas susceptibles al cambio climático.

\section{MATERIALES Y MÉTODOS}

\section{Área de estudio}

El Eje Neovolcánico Transversal se localiza entre las coordenadas geográficas $17^{\circ} 30^{\prime}$ y $20^{\circ} 25^{\prime}$ latitud norte y $96^{\circ} 20^{\prime}$ y $105^{\circ} 20^{\prime}$ longitud oeste y tiene una longitud de 930 km (Demant y Vincent, 1978) (Fig. 1).

\section{Datos de presencia}

Se descargaron 247 registros (coordenadas GPS) disponibles de Pinus hartwegii Lindl, para México, de la aplicación Niche ToolBox (Osorio-Olvera, Vijay, Narayani, Soberón y Falconi, 2018), de la Comisión Nacional para el Uso y Conocimiento de la Biodiversidad (Conabio) y se obtuvieron 96 registros GPS de la especie en campo.

Utilizando la aplicación de Niche Toolbox, se suprimieron las coordenadas duplicadas y con una distancia de separación menor a $1 \mathrm{~km}$ entre cada uno de los registros, dejando así, una sola coordenada por cada celda de $1 \mathrm{~km}^{2}$. Este paso ayudó a evitar la sobreestimación de los modelos por los efectos de la autocorrelación espacial (Peterson y Nakazawa, 2008; Monterrubio-Rico et al., 2016). Se dejaron solo los registros ubicados dentro del Eje Neovolcánico Transversal y se eliminaron aquellos ubicados en áreas urbanas y por debajo de los $3000 \mathrm{~m}$ snm, que es la altitud 


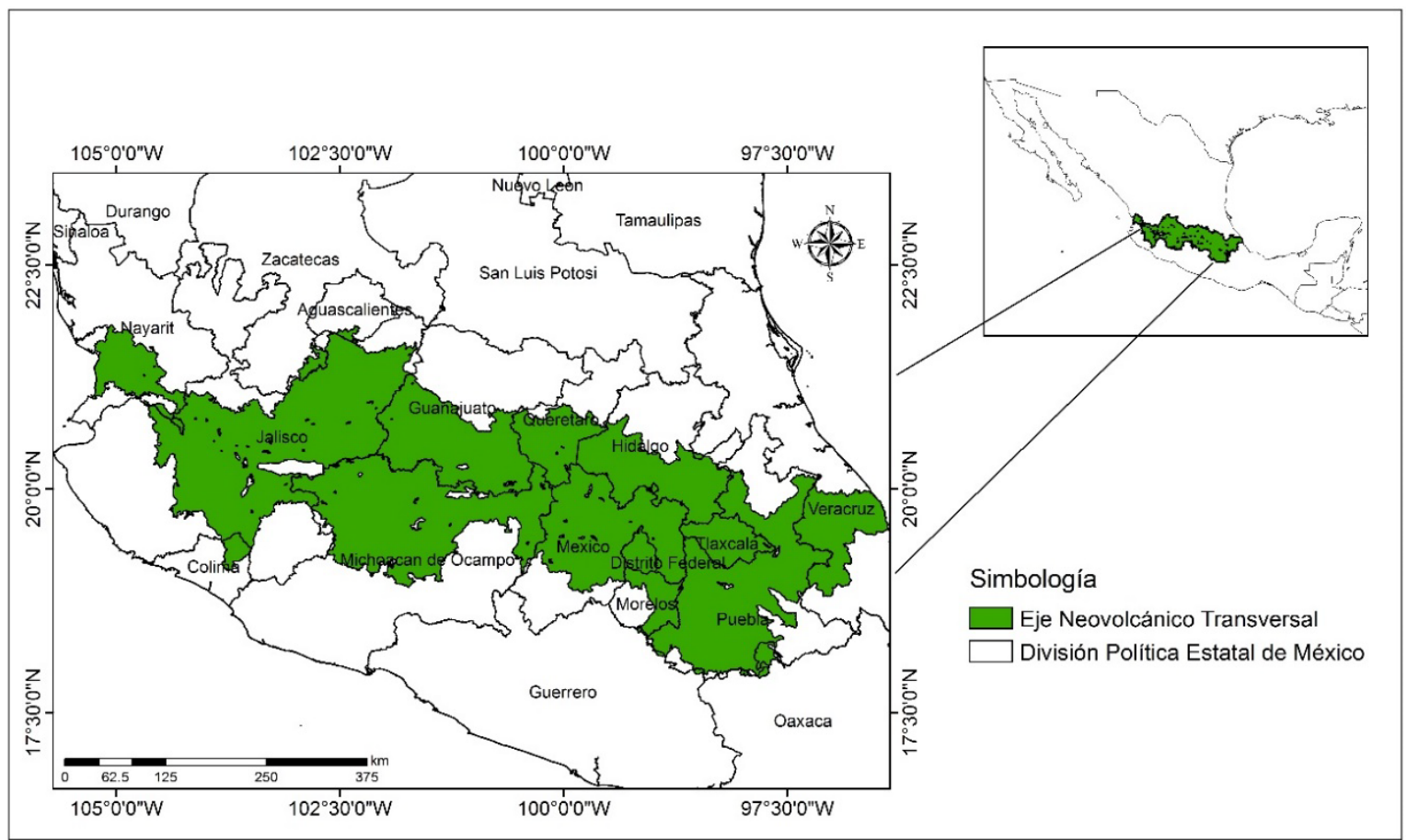

FIGURA 1. Área de estudio

señalada por Farjon et al. (1997), donde la especie forma bosques puros. Al final del proceso de depuración, se obtuvo una base de datos con un total de 65 registros para Pinus hartwegii Lindl; 60 de ellos fueron extraídos de la plataforma GBIF a través de la aplicación Niche Toolbox y cinco obtenidos en campo.

\section{Obtención de información bioclimática actual y futura}

Se descargaron las 19 variables bioclimáticas de Worldclim ver. 2.0 para el periodo presente (1970-2000) (Fick y Hijmans, 2017) y futuro de los modelos de circulación global CRNMCM5 (Centro Nacional de Investigaciones Meteorológicas de Francia) y HADGEM2_ES (Centro Meteorológico Hadley del Reino Unido). Estos MCG fueron generados a partir de modelos regionales del proyecto de intercomparación de modelos acoplados fase 5 (CMIP5) con dos forzamientos radiactivos de 4.5 (trayectorias de $\mathrm{CO}_{2}$ constantes) y 8.5 (trayectorias de $\mathrm{CO}_{2}$ altas) para 2041-2060, tienen una resolución espacial de 1 $\mathrm{km}^{2} \mathrm{y}$ un formato GeoTiff (Tiff) (Worldclim, 2018).
La tabla 1 describe las 19 variables bioclimáticas descargadas para el presente y futuro.

\section{Selección de variables}

Debido a que las 19 variables bioclimáticas descargadas están correlacionadas entre sí, se procedió a eliminar la autocorrelación espacial mediante un análisis de multicolinearidad (Peterson y Nakazawa, 2008; Monterrubio-Rico et al., 2016). La aplicación de dicho análisis ayudó a descartar aquellas variables con un coeficiente de correlación mayor o igual a 0.85 , lo cual permitió maximizar la contribución de las variables en los modelos de distribución (Peterson y Nakazawa, 2008; Ortego, Gugger, Riordan y Sork, 2014; Martínez-Méndez, Aguirre-Planter, Eguiarte y Jaramillo-Correa, 2016; Monterrubio-Rico et al., 2016). Se incluyeron también las variables pendiente (PEN; 1 km de resolución), suelo (SUE; escala 1; 250000), vegetación (VEG; serie V del Instituto Nacional de Estadística y Geografía [Inegi], escala 1; 250000) y altitud (DEM; modelo digital de elevación, resolución de $90 \mathrm{~m}$ ) las cuales fueron descargadas en 
TABLA 1. Variables bioclimáticas para los periodos presente y futuro

\begin{tabular}{|c|c|}
\hline Variables bioclimáticas (Unidades entre paréntesis) & Clave \\
\hline Temperatura promedio anual $\left({ }^{\circ} \mathrm{C}\right)$ & $\mathrm{BIO1}$ \\
\hline Oscilación diurna de la temperatura $\left({ }^{\circ} \mathrm{C}\right)$ & $\mathrm{BIO} 2$ \\
\hline Isotermalidad (BIO2/BIO7)(*100) & $\mathrm{BIO3}$ \\
\hline Estacionalidad de la temperatura (Coeficiente de variación, \%) & $\mathrm{BIO4}$ \\
\hline Temperatura máxima promedio del periodo más cálido $\left({ }^{\circ} \mathrm{C}\right)$ & $\mathrm{BIO5}$ \\
\hline Temperatura mínima promedio del periodo más frío $\left({ }^{\circ} \mathrm{C}\right)$ & $\mathrm{BIO6}$ \\
\hline Oscilación anual de la temperatura $\left({ }^{\circ} \mathrm{C}\right)$ & $\mathrm{BIO7}$ \\
\hline Temperatura promedio del trimestre más lluvioso $\left({ }^{\circ} \mathrm{C}\right)$ & $\mathrm{BIO8}$ \\
\hline Temperatura promedio del trimestre más seco $\left({ }^{\circ} \mathrm{C}\right)$ & BIO9 \\
\hline Temperatura promedio del trimestre más cálido $\left({ }^{\circ} \mathrm{C}\right)$ & $\mathrm{BIOIO}$ \\
\hline Temperatura promedio del trimestre más frío $\left({ }^{\circ} \mathrm{C}\right)$ & BIOl1 \\
\hline Precipitación acumulada anual (mm) & $\mathrm{BIO12}$ \\
\hline Precipitación del periodo más lluvioso (mm) & $\mathrm{BIO13}$ \\
\hline Precipitación del periodo más seco (mm) & $\mathrm{BIO14}$ \\
\hline Estacionalidad de la precipitación (Coeficiente de variación, \%) & $\mathrm{BIO15}$ \\
\hline Precipitación del trimestre más lluvioso (mm) & $\mathrm{BIO16}$ \\
\hline Precipitación del trimestre más seco (mm) & $\mathrm{BIO17}$ \\
\hline Precipitación del trimestre más cálido (mm) & $\mathrm{BIO18}$ \\
\hline Precipitación del trimestre más frío (mm) & $\mathrm{BIO19}$ \\
\hline
\end{tabular}

formatos vectorial y raster de las plataformas de Conabio e Inegi (Conabio, 2016; Conabio-Inifap, 1995; Inegi, 2013; Inegi, 2018) y transformadas a archivos ASCII para integrarlas en la generación de los modelos de distribución.

\section{Delimitación del área $\mathrm{M}$}

El área de modelamiento espacial, $\mathrm{M}$, es el espacio geográfico y ambiental donde se ha registrado la presencia de una especie y se delimita de acuerdo con el conocimiento biológico que se tiene de ella y con su capacidad de dispersión (Soberón y Peterson, 2005; Martínez-Méndez et al., 2016). Aunado a este proceso, el uso de barreras biogeográficas en modelación espacial evita generar áreas de distribución sobreestimadas de la especie de interés (Martínez-Meyer, 2005).

Para delimitar el área $M$, se emplearon las subprovincias fisiográficas en donde se ubicaban los registros de presencia de la especie, las cuales fueron Lagos y Volcanes de Anáhuac, Neovolcánica Tarasca y Volcanes de Colima (Inegi, 2001). El área M sirvió para recortar las variables ambientales a un mismo tamaño.

\section{Modelos de distribución de especies}

MaxEnt es un algoritmo que funciona mediante el principio de máxima entropía, el programa calcula la probabilidad de áreas idóneas para una especie a partir de coordenadas puntuales de presencia, no requiere datos de ausencia y genera buenas predicciones, aun cuando las muestras son pequeñas (Phillips, Anderson y Schaphire, 2006; Pearson, Raxworthy, Nakamura y Peterson, 2007).

Para generar los modelos de distribución de Pinus hartwegii Lindl se utilizaron los registros de presencia de la especie y las variables ambientales seleccionadas del análisis de multicolinearidad (BIO1, BIO2, BIO3, BIO4, BIO12, BIO15, BIO16, BIO18, PEN, SUE, VEG y DEM) en formato ASCII.

Estas variables se cargaron al programa MaxEnt Ver. 3.3.3, utilizando $75 \%$ de los registros de presencia seleccionados al azar para generar los modelos y $25 \%$ restante para validarlos (Phillips et al., 2006). Se desactivaron las casillas Extrapolate y Do clamping con el fin de evitar sobreajustar los modelos (Elith et al., 2011), la salida de los modelos fue del tipo logística.

Se generaron y probaron 10 modelos de distribución con el fin de determinar, por medio de las pruebas estadísticas del área bajo la curva (ABC), Roc parcial y Z, cuál modelo explica mejor la distribución actual de la especie. Dichos modelos se construyeron a partir de diversos criterios, los cuales se especifican en la tabla 2; siendo M5 y M10, los modelos generados a partir de los 
parámetros de omisión del programa (Phillips et al., 2006) pero con diferente replicado interno.

\section{Validación de los modelos de distribución}

Los modelos se evaluaron por medio de los valores del área bajo la curva $(\mathrm{ABC})$ del análisis de las características operativas del receptor (ROC). Los valores de esta prueba oscilan de 0 a 1: valores entre 0.7 y 0.9 clasifican el desempeño de los modelos como bueno y valores mayores a 0.9 los califican como excelente (Peterson et al., 2011; Coitiño, Montenegro, Fallabrino, González y Hernández, 2013); sin embargo, esta validación ha sido cuestionada por no considerar datos de ausencias verdaderas (Peterson, Papes y Soberón, 2008).

Se utilizó el programa Tool for Partial-ROC versión 1.0 (Narayani, 2008). Donde los dos subconjuntos de datos (presencia e idoneidad de hábitat) fueron contrastados con el modelo reclasificado en probabilidades de MaxEnt para obtener los valores puntuales de adecuación o idoneidad. También se extrajo el número de pixeles de cada valor de probabilidad del modelo reclasificado. Los archivos preparados en formato CSV se ingresaron al programa Tool for Partial-ROC ver. 1.0. Para el análisis, se usó una proporción de puntos para Bootraps de 50\%, con un número de muestras con reemplazo de 1000 réplicas y un umbral de omisión de 95\%. Se promedió el valor de radio de las 1000 réplicas de los modelos. Los valores de esta prueba oscilan de 1 a 2: un valor con promedio de radio de 1 representa un modelo igual al azar (Peterson et al., 2008; Martínez-
Méndez et al., 2016; Garza-López et al., 2016). Se realizó la prueba de hipótesis $z$.

El modelo de distribución con el mejor ajuste para Pinus hartwegii Lindl se eligió con base en el valor más alto del análisis Roc parcial, menor error estándar y valor significativo de $z$. Los valores de idoneidad del modelo con el mejor ajuste estadístico se reclasificaron a un modelo de calidad de hábitat de tres categorías con intervalos iguales: baja, moderada y alta. Se utilizó el valor de la categoría de calidad de hábitat alta (> 0.60); con ayuda del módulo reclass del programa Arcmap 10.3® (Environmental Systems Research Institute [ESRI], 2014), el modelo se transformó de continuo a binario (apto-no apto), dicha transformación ayudó a calcular y delimitar la distribución actual del bosque subalpino puro de Pinus hartwegii Lindl en el Eje Neovolcánico Transversal.

\section{Modelación bajo escenarios de cambio climático}

Para generar los modelos de distribución bajo escenarios de cambio climático, se transfirieron los parámetros del modelo de distribución actual con el mejor ajuste estadístico (Morrone y Escalante, 2016) al programa MaxEnt Ver. 3.3.3. Se cargaron las variables ambientales en formato ASCII y los registros de la especie en formato CSV. Se utilizaron $75 \%$ de los registros de la especie para generar los modelos y 25\% restante para validarlos (Phillips et al., 2006), se desactivaron las opciones Extrapolate y Do clamping con el fin de evitar sobreajustar los modelos de distribución (Elith et al., 2011).

TABLA 2. Criterios empleados en la generación de los modelos de distribución

\begin{tabular}{llll}
\hline Modelo & Replicado & Regla de aplicación de umbral & Réplicas \\
\hline M1 & Bootraps & Sensibilidad y especificidad de entrenamiento iguales & 1000 \\
M2 & Bootraps & Máxima sensibilidad de entrenamiento más especificidad & 1000 \\
M3 & Bootraps & Prueba de sensibilidad y especificidad iguales & 1000 \\
M4 & Bootraps & Prueba de máxima sensibilidad más especificidad & 1000 \\
M5 & Bootraps & Sin aplicación de regla de umbral & 500 \\
M6 & Validación Cruzada & Sensibilidad y especificidad de entrenamiento iguales & 1000 \\
M7 & Validación Cruzada & Máxima sensibilidad de entrenamiento más especificidad & 1000 \\
M8 & Validación Cruzada & Prueba de sensibilidad y especificidad iguales & 1000 \\
M9 & Validación Cruzada & Prueba de máxima sensibilidad más especificidad & 1000 \\
M10 & Validación Cruzada & Sin aplicación de regla de umbral & 500 \\
\hline
\end{tabular}




\section{Validación de los modelos bajo escenarios de cambio climático}

Los modelos fueron evaluados por medio de los valores ABC del análisis ROC (Phillips et al., 2006) y las pruebas de Roc parcial (Narayani, 2008) y de Z (Monterrubio-Rico et al., 2016). Se reclasificaron los valores de idoneidad en tres categorías; baja, moderada y alta y se utilizó el valor de la categoría de calidad de hábitat alta $(>0.60)$ como umbral para convertir los modelos continuos a binarios (apto-no apto).

\section{Variables determinantes en la distribución actual y futura}

Por medio de la prueba de Jackknife se determinó la contribución de las variables en la generación de los modelos de distribución (Phillips et al., 2006). Dicha prueba permitió identificar los requerimientos ambientales adecuados para que Pinus hartwegii Lindl crezca, se desarrolle y subsista en la actualidad y a futuro en el Eje Neovolcánico Transversal.

\section{Escenarios de cambio climático}

De acuerdo con el forzamiento radiactivo empleado, los escenarios de cambio climático simulan la posible afectación de un conjunto de variables ambientales en la distribución de una especie. Las trayectorias se clasifican en conservadoras (RCP 4.5; trayectorias de $\mathrm{CO}_{2}$ constantes) y extremas (RCP 8.5; trayectorias de $\mathrm{CO}_{2}$ altas), las primeras predicen un aumento en temperatura promedio anual de 0.9 ${ }^{\circ} \mathrm{C}$ a $2{ }^{\circ} \mathrm{C}$ y las segundas un incremento de $1.4{ }^{\circ} \mathrm{C}$ a $2.6{ }^{\circ} \mathrm{C}$ en la temperatura promedio anual global para el 2050 (IPCC, 2013; Fernández-Eguiarte et al., 2015). Los escenarios de cambio climático referencia a una pérdida menor de superficie con las condiciones aptas para que la especie crezca y sobreviva, mientras que los escenarios extremos predicen pérdidas mayores en la distribución potencial de una especie.

Utilizando la información vectorial disponible de las áreas naturales protegidas federales (ANP) de la Conanp (2017) y sobreponiendo los archivos de las salidas binarias de los modelos actual-futuro con mejor desempeño estadístico en el programa Arcmap 10.3® (ESRI, 2014), fue posible identificar las áreas susceptibles y estimar la distribución reducida por efectos del cambio climático en la poligonal de las ANP del área de estudio.

\section{ResUltadOS}

Los 10 modelos de distribución actual presentaron valores de 0.986 para los datos de entrenamiento y 0.969 para los de validación en el $\mathrm{ABC}$ de la prueba de ROC, lo que indica que el desempeño de los modelos fue excelente. Los valores de las pruebas de Roc parcial y Z obtuvieron un ajuste estadístico excelente (Tabla 3).

De los 10 modelos probados, el 7 presentó el mejor ajuste estadístico. Este modelo fue generado mediante un replicado interno tipo validación cruzada, regla de aplicación de umbral de máxima sensibilidad de entrenamiento más especificidad y 1000 réplicas.

De acuerdo con la reclasificación de los valores de idoneidad de la calidad de hábitat alta del modelo 7, la superficie actual de bosque subalpino puro de Pinus hartwegii Lindl es de 34717 ha (Fig. 2).

\section{Modelación bajo escenarios de cambio climático}

Los valores del $\mathrm{ABC}$ de la prueba de ROC fueron de 0.983 a 0.984 para el entrenamiento y de 0.960 a 0.970 para la validación, lo cual indica que el desempeño de los modelos fue excelente. Los resultados de las pruebas de Roc parcial y $\mathrm{Z}$ presentaron un desempeño estadístico excelente (Tabla 4).

TABLA 3. Desempeño estadístico Roc parcial y Z de los modelos de distribución actual

\begin{tabular}{llll}
\hline Modelo & $\begin{array}{l}\text { Roc parcial } \\
\text { promedio radios }\end{array}$ & $\begin{array}{l}\text { Error } \\
\text { estándar }\end{array}$ & Prueba de Z \\
\hline M1 & 1.895 & 0.014 & $\mathrm{P}<0.01$ \\
M2 & 1.894 & 0.013 & $\mathrm{P}<0.01$ \\
M3 & 1.894 & 0.014 & $\mathrm{P}<0.01$ \\
M4 & 1.894 & 0.014 & $\mathrm{P}<0.01$ \\
M5 & 1.895 & 0.014 & $\mathrm{P}<0.01$ \\
M6 & 1.895 & 0.014 & $\mathrm{P}<0.01$ \\
M7 & 1.895 & 0.013 & $\mathrm{P}<0.01$ \\
M8 & 1.895 & 0.014 & $\mathrm{P}<0.01$ \\
M9 & 1.895 & 0.014 & $\mathrm{P}<0.01$ \\
M10 & 1.894 & 0.014 & $\mathrm{P}<0.01$ \\
\hline
\end{tabular}




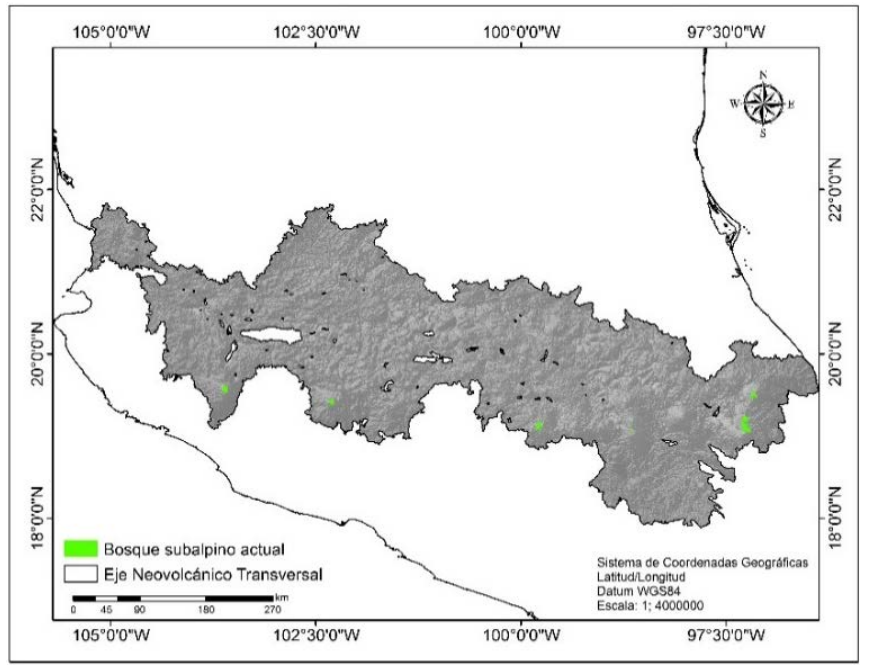

Figura 2. Distribución actual del bosque subalpino de Pinus hartwegii Lindl en el Eje Neovolcánico Transversal

Los resultados de las pruebas de Roc parcial, error estándar y $\mathrm{Z}$ indican un mejor ajuste en las trayectorias RCP para el modelo de circulación global CRNMCM5 en comparación con las trayectorias del modelo HADGEM2_ES.

\section{Variables determinantes en la distribución actual y futura}

Las variables ambientales relevantes actuales fueron BIO1, BIO18, BIO12, SUE y VEG, las cuales aportaron $84.9 \%$ de la variabilidad de los modelos (Fig. 3).

Las variables ambientales relevantes para 2041-2060, de acuerdo con el modelo CRNMCM5 para las trayectorias RCP 4.5 y 8.5, fueron BIO1, SUE, BIO16, PEN y VEG, con una contribución de $94.2 \%$ y $92.6 \%$, respectivamente (Fig. 4).

Por otra parte, las variables relevantes para 2041-2060 para las trayectorias RCP 4.5 y 8.5 del modelo HADGEM2_ES fueron DEM, BIO1, SUE, VEG y BIO12, con una contribución de $88.1 \%$ y $88.5 \%$, respectivamente (Fig. 5).
Los resultados de los modelos de distribución señalan a la BIO1 y DEM como las variables determinantes en la distribución actual y futura del bosque subalpino de Pinus hartwegii Lindl en el Eje Neovolcánico Transversal.

\section{Escenarios de cambio climático}

Los resultados de la trayectoria RCP 4.5 prevén un aumento en temperatura promedio anual entre $0.7^{\circ} \mathrm{C}$ (CRNMCM5) y $1.5{ }^{\circ} \mathrm{C}$ (HADGEM2_ES), dichos aumentos podrían provocar reducciones entre $11.7 \%$ (CRNMCM5) y $18.3 \%$ (HADGEM2_ES) en la distribución actual del bosque subalpino de Pinus hartwegii Lindl en el Eje Neovolcánico Transversal para 2041-2060.

Los resultados de la trayectoria RCP 8.5 predicen un aumento en temperatura promedio anual entre $1.1{ }^{\circ} \mathrm{C}$ (CRNMCM5) y $2.1{ }^{\circ} \mathrm{C}$ (HADGEM2_ES); ante tales aumentos en temperatura promedio anual, la distribución actual del bosque subalpino de Pinus hartwegii Lindl se vería reducida en $18.3 \%$ (CRNMCM5) hasta $26.9 \%$ (HADGEM2_ES) para 2041-2060.

En la tabla 5 se describe la superficie actual y la estimada para el bosque subalpino de Pinus hartwegii Lindl de los dos modelos de circulación global probados para 2041-2060.

Debido a la gran escala del área de estudio, esta se dividió en cuatro zonas, las cuales abarcan la distribución subalpina actual y futura de Pinus hartwegii Lindl en el Eje Neovolcánico Transversal. La zona 1 abarca las áreas naturales protegidas Volcán Nevado de Colima, la zona 2 el Pico de Tancítaro, la zona 3 comprende el Nevado de Toluca e Izta-Popo y la zona 4 está conformada por La Malinche, El Cofre de Perote y El Pico de Orizaba. La clasificación del área de estudio por zonas permitió identificar las áreas naturales protegidas más susceptibles a los efectos del cambio climático.

TABLA 4. Resultados Roc parcial y Z de los modelos para 2041-2060

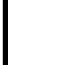

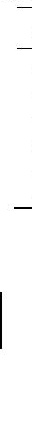

Modelo

CRNMCM5 RCP 4.5

HADGEM2_ES RCP 4.5

CRNMCM5 RCP 8.5

HADGEM2_ES RCP 8.5

Roc parcial promedio radios

1.895

1.546

1.906

1.401

$\begin{array}{ll}\text { Error estándar } & \text { Prueba de Z } \\ 0.019 & \mathrm{P}<0.01 \\ 0.246 & \mathrm{P}<0.01 \\ 0.016 & \mathrm{P}<0.01 \\ 0.247 & \mathrm{P}<0.01\end{array}$




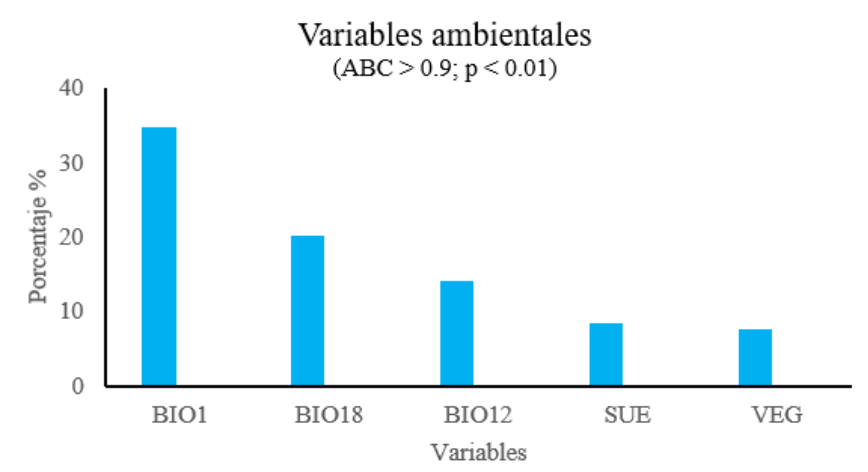

FIGURA 3. Variables importantes en los modelos actuales

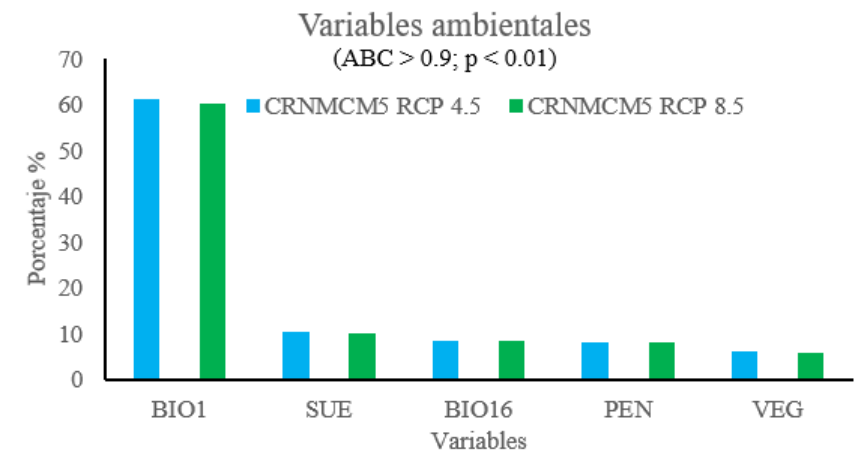

FIGURA 4. Variables importantes del modelo CRNMCM5 a futuro

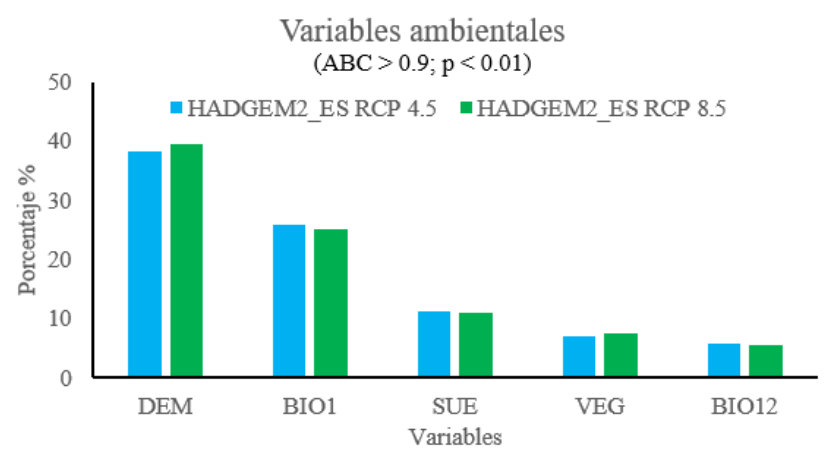

FIGURA 5. Variables importantes del modelo HADGEM2_ES a futuro

TABLA 5. Superficie actual y futura del bosque subalpino de Pinus hartwegii Lindl

\begin{tabular}{ll}
\hline Modelo & Superficie (ha) \\
\hline Actual & 34717 \\
CRNMCM5 RCP 4.5 & 30662 \\
HADGEM2_ES RCP 4.5 & 26701 \\
CRNMCM5 RCP 8.5 & 28348 \\
HADGEM2_ES RCP 8.5 & 25392 \\
\hline
\end{tabular}

La tabla 6 muestra las superficies actuales y futuras estimadas por zonas para el bosque subalpino de Pinus hartwegii Lindl en el Eje Neovolcánico Transversal.

En la tabla 6 se puede observar que los modelos bajo escenarios de cambio climático predicen que las zonas $3 \mathrm{y}$ 4 serían las más afectadas por los efectos del cambio climático a futuro.

La figura 6 muestra la distribución de las condiciones ambientales idóneas actual y futura de los escenarios de cambio climático conservador (CRNMCM5 RCP 4.5) para el bosque subalpino de Pinus hartwegii Lindl, dentro de la poligonal de las áreas naturales protegidas del Eje Neovolcánico Transversal.

De acuerdo con los resultados del escenario climático conservador (MCG CRNMCM5 RCP 4.5) para 2041-2060, los sitios más susceptibles al cambio climático serían el Nevado de Toluca, con pérdidas de hasta 53.1\% (2853.5 ha), el Pico de Orizaba con una reducción de hasta 33.5\% (3292.1 ha) y La Malinche con una disminución de hasta 19.6\% (54.3 ha) en la distribución actual de Pinus hartwegii Lindl. Sitios como el Volcán Nevado de Colima (+1.9\%, 61 ha) y el Cofre de Perote $(+3.5 \%, 160.8$ ha) presentarían un aumento en la superficie idónea, mientras que el Pico de Tancítaro presentaría una ligera reducción $(0.7 \%, 27.2 \mathrm{ha})$. Estos resultados indican que estos sitios no serían tan afectados por el cambio climático; sin embargo, dichas modificaciones sí cambiarían sus distribuciones para 20412060. Por otra parte, el Izta-Popo presentaría un aumento de hasta 37.4\% (361.5 ha) para 2041-2060.

\section{DISCUSIÓN}

Los diversos estudios realizados sobre modelamiento de distribución de especies de la familia Pinaceae bajo escenarios de cambio climático para México concuerdan en la teoría de una reducción importante en la distribución a futuro de esas especies (Sáenz-Romero et al., 2012, 2015; Estrada-Contreras et al., 2015; Cruz-Cárdenas et al., 2016). El presente estudio es el primero en delimitar, determinar las variables ambientales importantes, estimar y evaluar los efectos del cambio climático en la distribución actual y 
futura en el bosque subalpino de Pinus hartwegii Lindl en el Eje Neovolcánico Transversal.

Los resultados de las pruebas de ROC, Roc Parcial y Z de los modelos de distribución presentaron un desempeño excelente (Peterson et al., 2008; Peterson et al., 2011; Monterrubio-Rico et al., 2016), la combinación de los parámetros en modelación permitió determinar el modelo (modelo 7 [validación cruzada, regla de aplicación de umbral de Máxima sensibilidad de entrenamiento más especificidad y 1000 réplicas]) con el mejor ajuste estadístico y con el cual fue posible estimar y delimitar las distribuciones actual y futura, bajo escenarios de cambio climático (2041-2060) para el bosque subalpino de Pinus bartwegii Lindl en el Eje Neovolcánico Transversal.

TABLA 6. Superficies actuales y futuras por zonas para el bosque subalpino de Pinus hartwegii Lindl en el Eje Neovolcánico

Transversal

\begin{tabular}{lllll}
\hline Modelo de distribución & $\begin{array}{l}\text { Superficie ha } \\
\text { Zona 1 }\end{array}$ & $\begin{array}{l}\text { Superficie ha } \\
\text { Zona 2 }\end{array}$ & $\begin{array}{l}\text { Superficie ha } \\
\text { Zona 3 }\end{array}$ & $\begin{array}{l}\text { Superficie ha } \\
\text { Zona 4 }\end{array}$ \\
\hline Actual & 4984.2 & 4045.2 & 6338 & 19349.6 \\
CRNMCM5 RCP 4.5 & 3230.5 & 5232.6 & 5037.9 & 17161 \\
HADGEM2_ES RCP 4.5 & 3940 & 5600 & 5478 & 13330 \\
CRNMCM5 RCP 8.5 & 4687 & 4128.8 & 4153.2 & 13732 \\
HADGEM2_ES RCP 8.5 & 4256 & 4093 & 3885 & 13158 \\
\hline
\end{tabular}
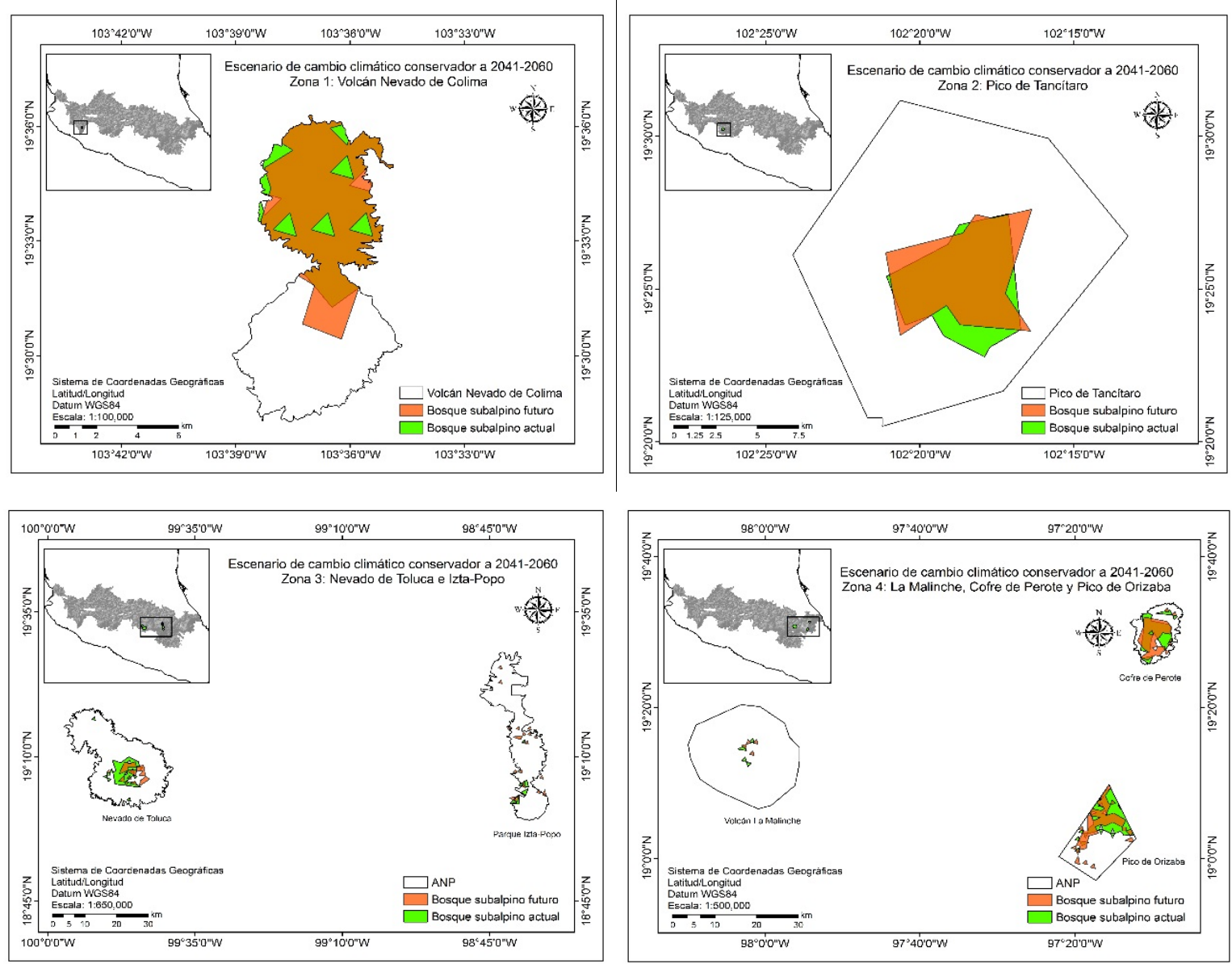

FIgura 6. Distribución actual y futura del escenario conservador (CRNMCM5 RCP 4.5) para el bosque subalpino de Pinus hartwegii Lindl en el Eje Neovolcánico Transversal. 
Trabajos como los de Martínez-Méndez et al. (2016) y Garza-López et al. (2016) mencionan que el funcionamiento de los modelos de distribución mejora cuando se aplican diferentes criterios de modelación. En el presente trabajo, los modelos generados presentaron un funcionamiento similar en las pruebas de ROC y Roc parcial, solamente fue posible diferenciar el modelo con el mejor desempeño a través del error estándar.

\section{Variables determinantes para Pinus hartwegii Lindl}

Las variables relevantes para ambos periodos analizados fueron BIO1, DEM, BIO18, BIO12, SUE y VEG. Una oscilación de la temperatura promedio anual de $6.8^{\circ} \mathrm{C}$ a $13.1^{\circ} \mathrm{C}$ es importante en el crecimiento y desarrollo de la especie, estos resultados son ligeramente menores a los obtenidos por Gutiérrez y Trejo (2014), quienes mencionan que la temperatura promedio anual para el crecimiento de Pinus hartwegii Lindl es de $9.9^{\circ} \mathrm{C}$ a $13{ }^{\circ} \mathrm{C}$. También resultan ser menores a los obtenidos por Martínez-Méndez et al. (2016) para Abies religiosa (Kunth) Schltdl \& Cham $\left(12{ }^{\circ} \mathrm{C}\right.$ a $16.4^{\circ} \mathrm{C}$ ), especie que coexiste con Pinus hartwegii Lindl en las partes altas del Eje Neovolcánico Transversal.

Martínez-Méndez et al. (2016) mencionan que la DEM es un factor importante en la distribución de cinco especies de abetos mexicanos (Abies religiosa, Abies concolor, Abies guatemalensis, Abies bickelii y Abies durangensis var. coahuilensis), al igual que para estas especies, la altitud es un factor importante para la distribución de Pinus hartwegii Lindl en el Eje Neovolcánico Transversal.

Para la BIO18, los mismos autores del párrafo anterior encontraron que esta variable es relevante en la distribución de cinco especies de abetos mexicanos (Abies concolor, Abies guatemalensis, Abies durangensis var. durangensis, Abies durangensis var. coahuilensis y Abies vejarii). Sáenz-Romero et al. (2012) mencionan que la precipitación de los meses de verano es importante en la distribución actual de Abies religiosa en el Eje Neovolcánico Transversal. Por lo que la variable (BIO18) es determinante en la distribución actual de especies de la familia Pinaceae que se localizan en las partes altas de México. De acuerdo con los resultados obtenidos en este estudio, los valores adecuados de la precipitación durante los meses de verano para el crecimiento de Pinus hartwegii Lindl son de $316 \mathrm{~mm}$ a 575 $\mathrm{mm}$.

La precipitación total anual idónea para el desarrollo de Pinus hartwegii Lindl es de 1304 mm; de acuerdo con los resultados de los modelos a futuro, se prevé una disminución entre $2.8 \%$ y $3.8 \%$ en la precipitación promedio anual para 2041-2060. Estos resultados difieren de los estimados por Sosa-Rodríguez (2015) y SáenzRomero et al. (2010), quienes mencionan que la precipitación promedio anual en México disminuiría hasta $20 \%$ para 2020 y 9\% para 2060. Manzanilla y Aguirre (2017) estimaron una disminución de $2.6 \%$ a $5.3 \%$ en la precipitación promedio anual de la reserva de la biosfera Selva el Ocote, Chiapas para 2030; resultados similares a los obtenidos en el presente estudio.

Para la variable SUE, trabajos como el Cruz-Cárdenas et al. (2016) mencionan que el $\mathrm{pH}$ del suelo es importante en la distribución de seis especies de pináceas en el estado de Michoacán, pero no sustancial para Pinus hartwegï Lindl, aunque para este trabajo, el suelo fue ligeramente importante. Estudios como el de Galicia et al. (2016) sugieren que las pináceas del centro de México prefieren suelos del tipo Andosol, los cuales son ligeramente ácidos. En este trabajo, las zonas 2, 3 y 4 presentaron suelos del tipo Andosol.

En cuanto a la VEG, de acuerdo con Inegi (2013) y con lo obtenido en este trabajo, el bosque subalpino de Pinus hartwegii Lindl del Eje Neovolcánico Transversal coincide con los tipos de vegetación pradera alta de montaña, bosque de pino y bosque de oyamel. Trabajos como los de Estrada-Contreras et al. (2015) e Islas-Báez et al. (2015) mencionan que los tipos de vegetación son importantes en la distribución potencial actual y futura de una especie.

Bajo el contexto de protección legal de especies de la familia Pinaceae en México, estudios como el de Aguirre y Duivenvoorden (2010) mencionan que 70\% de los 56 taxa 
del género Pinus modelados en México tienen solo 10\% de su distribución natural resguardaba dentro de la poligonal de alguna área natural protegida federal.

Por otra parte, Martínez-Méndez et al. (2016) hallaron que nueve de los diez taxa del género Abies registrados en México presentan su área de distribución fuera la poligonal de las áreas naturales protegidas. En el presente trabajo, el bosque subalpino actual de Pinus hartwegii Lindl se encuentra protegido en $86.5 \%$ por las áreas naturales protegidas que se encuentran en el Eje Neovolcánico Transversal. Sin embargo, dichas áreas no están exentas de los efectos del cambio climático. Los modelos a futuro prevén que la temperatura promedio anual en el bosque de Pinus hartwegii Lindl aumente entre $0.7^{\circ} \mathrm{C}$ y $2.1^{\circ} \mathrm{C}$ por lo que, de acuerdo con el IPCC (2014), aumentos de $1{ }^{\circ} \mathrm{C}$ a $2{ }^{\circ} \mathrm{C}$ representarían un daño moderado a la biodiversidad y arriba de $3.5^{\circ} \mathrm{C}$ sería perjudicial para muchas especies.

Garza-López et al. (2016), utilizando trayectorias RCP de 6.0 (promedio de 18 Modelos de Circulación Global), estimaron una reducción de $60 \%$ en el hábitat climático de la caoba (Swietenia macrophylla) en la Península de Yucatán para 2030. En este trabajo, se prevén reducciones entre 11.7\% (escenario RCP 4.5 MCG CRNMCM5) y $26.9 \%$ (escenario RCP 8.5 MCG HADGEM2_ES) en la distribución actual de Pinus hartwegii Lindl. Siendo el Nevado de Toluca y el Pico de Orizaba los sitios más susceptibles al cambio climático de acuerdo con los modelos proyectados a futuro.

Villers y Trejo (1998) y Sáenz-Romero et al. (2012) mencionan que los bosques secos aumentarían su distribución (7.4\%), mientras que los bosques templados y fríos reducirían sus nichos ecológicos entre $65 \%$ y $87.6 \%$, respectivamente; además, sitios como la reserva Mariposa Monarca presentarían condiciones más cálidas $\left(2.3^{\circ} \mathrm{C}\right)$ para 2060 (Sáenz-Romero et al., 2010), condiciones climáticas similares a las que se llegarían a presentar, de acuerdo con los resultados obtenidos en este trabajo $\left(0.7^{\circ} \mathrm{C}\right.$ a $\left.2.1^{\circ} \mathrm{C}\right)$ para 2041-2060 para la misma región geográfica. SáenzRomero et al., 2010 mencionan para que una especie arbórea pueda subsistir a los cambios en el clima deberá migrar altitudinalmente $100 \mathrm{~m}$ por cada $0.5^{\circ} \mathrm{C}$ de aumento. Los resultados del presente trabajo sugieren un aumento en temperatura promedio anual entre $0.7{ }^{\circ} \mathrm{C}$ y $2.1^{\circ} \mathrm{C}$ para 2041-2060; antes tales cambios, Pinus hartwegii Lindl estaría obligado a desplazarse entre $100 \mathrm{~m}$ y $500 \mathrm{~m}$ arriba, por lo que los bosques puros de la especie tendrían que reubicarse entre $3100 \mathrm{~m}$ y $3500 \mathrm{~m}$ snm y no en $3000 \mathrm{~m}$ snm, que señala actualmente Farjon et al. (1997).

Sáenz-Romero et al. (2012) indican que el aumento en temperatura media del mes más cálido reduciría en $87.6 \%$ la cubierta forestal de Abies religiosa para 2060 en el Eje Neovolcánico Transversal; por otra parte, el aumento en temperatura promedio anual para 2050 reduciría hasta $26.9 \%$ de la superficie de Pinus hartwegii Lindl para esa misma región geográfica.

Estrada-Contreras et al. (2015) hallaron un aumento de nicho de $3.7 \%$ para Veracruz para 2050, resultado similar a lo obtenido en este estudio para el Cofre de Perote (+3.5\%) para 2041-2060. Cruz-Cárdenas et al. (2016) prevén una reducción de 38.3\% en la superficie de Pinus hartwegii Lindl en Michoacán para el horizonte 2015-2039, resultado que es menor a lo obtenido en el presente estudio para el Nevado de Toluca (53.1\%). Islas-Báez et al. (2015) mencionan que la variable más importante en la distribución de Abies religiosa para 2030 y 2050 en la Reserva de la Biosfera Mariposa Monarca es la precipitación anual, variable que difiere con las variables determinantes (BIO1 y DEM) obtenidas de los modelos generados para este estudio.

De acuerdo con lo analizado en los trabajos anteriores y el presente estudio, los escenarios de cambio climático predicen panoramas no muy alentadores para el bosque subalpino de Pinus hartwegii Lindl en el Eje Neovolcánico Transversal. Ante la posible pérdida en la superficie boscosa subalpina, una de las consecuencias inmediatas en dicho ecosistema sería la reducción y función de los servicios ecosistémicos como la captura de carbono, recarga de acuíferos y regulación del clima local, lo cual afectaría directamente a las poblaciones rurales aledañas a los ecosistemas de alta montaña del centro de México. 


\section{CONCLUSIONES}

La principal aportación del presente trabajo es la estimación y delimitación actual y futura (2041-2060) de la distribución del bosque subalpino de Pinus hartwegii Lindl y el efecto del cambio climático en este tipo de ecosistema, el cual ha sido poco estudiado en México.

El bosque subalpino actual de Pinus hartwegii Lindl se encuentra bien resguardado por la red de áreas naturales protegidas federales de la Conanp, sin embargo, dicha protección no evitaría los efectos del cambio climático a futuro.

Las variables determinantes en la distribución actual y futura del bosque subalpino de Pinus hartwegii Lindl en el Eje Neovolcánico Transversal fueron la temperatura promedio anual y la altitud. Siendo estas dos variables las más relevantes para ambos periodos analizados.

Los escenarios de cambio climático para la especie no son muy alentadores, en ellos se predice una reducción de $11.7 \%$ a $26.9 \%$ en el nicho subalpino de Pinus hartwegii Lindl en el Eje Neovolcánico Transversal para 2041-2060.

Los sitios que se verían más afectados de acuerdo con la predicción de los modelos, serían el Nevado de Toluca y el Pico de Orizaba. Sin embargo, no todo sería reducción, sitios como el Parque Nacional Izta-Popo presentaría un aumento en su nicho de hasta $37.4 \%$.

Cabe mencionar que la reducción en las condiciones ambientales aptas para la especie no quiere decir que esta vaya a desaparecer completamente de esos sitios, por lo que es probable que crezca, pero con muchos factores limitantes.

Los resultados de este trabajo podrían ser útiles a los programas de adaptación y mitigación del cambio climático de los sitios analizados.

\section{RECONOCIMIENTOS}

Al Programa de Doctorado en Ciencias con Orientación en Manejo de Recursos Naturales de la Universidad Autónoma de Nuevo León, Facultad de Ciencias Forestales. Al Consejo Nacional de Ciencia y Tecnología (Conacyt) por el apoyo de beca de posgrado al primer autor. A los revisores anónimos por sus comentarios y sugerencias, los cuales sirvieron para mejorar el contenido del presente manuscrito.

\section{REFERENCIAS}

Aguirre G., J., \& Duivenvoorden, J. F. (2010). Can we expect to protect threatened species in protected areas? A case study of the genus Pinus in Mexico. Revista Mexicana de Biodiversidad, 81, 875-882. Recuperado de http://scielo.unam.mx/pdf/rmbiodiv/v81n3/v81n3a27.p df

Burgmer, T., Hillerbrand, H., \& Pfenninger, M. (2007). Effects of Climate-driven temperatura changes on the diversity of freshwater macroinvertebrates. Oecologia, 151(1), 93-103. doi: 10.1007/s00442-006-0542-9.

Coitiño, H. I., Montenegro, F., Fallabrino, A., González, E. M., \& Hernández, D. (2013). Distribución actual y potencial de Cabassous tatonay y Tamandua tetradactyla en el límite sur de su distribución: implicancias para su conservación en Uruguay. Edentata, 14(1), 23-34. doi: 10.5537/020.014.0104.

Comisión Nacional para el Uso y Conocimiento de la Biodiversidad [Conabio]-Instituto Nacional de Investigaciones Forestales y Agropecuarias [INIFAP] (1995). Edafología. Escala 1; 250000. Archivo vectorial Shapefile. Ciudad de México.

Comisión Nacional para el Uso y Conocimiento de la Biodiversidad [Conabio] (2016). Guevara, M. \& ArroyoCruz, C. E. Pendiente. Escala 1; 400000. Archivo raster Geotiff. Escala $1 \mathrm{~km}^{2}$. Ciudad de México.

Comisión Nacional de Áreas Naturales Protegidas [Conanp] (2015). Estrategia de cambio climático desde las Áreas Naturales Protegidas: Una Convocatoria para la Resiliencia de México (20152020) (1a ed.). México, D. F.: Secretaría de Medio Ambiente y Recursos Naturales.

Comisión Nacional de Áreas Naturales Protegidas [Conanp] (2017). Areas Naturales Protegidas Federales de México. Archivo vectorial Shapefile. Ciudad de México: Conanp.

Cruz-Cárdenas, G., López-Mata, L., Silva, J. T., Bernal-Santana, N., Estrada-Godoy, F., \& López-Sandoval, J. A. (2016). Potential distribution model of Pinaceae species under climate change scenarios in Michoacán. Revista Chapingo Serie Ciencias Forestales y del Ambiente, 22(2), 135-148. doi: 10.5154/r.rchscfa.2015.06.027.

Delgado, T., \& Suárez, D. D. (2009). Efectos del cambio climático en la diversidad vegetal del corredor de conservación comunitario Reserva Ecológica El Ángel bosque protector golondrinas en el norte del Ecuador. Ecología Aplicada, 8(2), 37-46. Recuperado de 
http://www.lamolina.edu.pe/ecolapl/Articulo_4_vol_8.pd f.

Demant, A., \& Vincent, P. M. (1978). A preliminary report on the comenditic dome and flow complex of Sierra La Primavera, Jalisco. Universidad Autónoma de México. Revista del Instituto de Geología, 2, 218-222.

Elith, J., Phillips, S. J., Hastie, T., Dudík, M., Chee, Y. E., \& Yates, C. J. (2011). A statistical explanation of MaxEnt for ecologist. Diversity and Distributions, 17(1), 43-57. doi: 10.1111/j.1472-4642.2010.00725.x

Environmental Systems Research Institute (ESRI). (2014). ArcGis Desktop: 10.3. Software diseñado para análisis espacial y Sistemas de Información Geográfica. Redlands, California, U.S.A.

Estrada-Contreras, I., Equihua, M., Castillo-Campos, G., \& Rojas-Soto, O. (2015). Climate change and effects on vegetation in Veracruz, Mexico: An approach using ecological niche modelling. Acta Botánica Mexicana, 112, 73 93. doi: $10.21829 / \mathrm{abm112} 2015.1090$

Galicia, L., Gamboa-Cáceres, A. M., Cram, S., Chávez-Vergara, B., Peña-Ramírez, V., Saynes, V., \& Siebe, C. (2016). Almacén y dinámica del carbono orgánico del suelo en bosques templados de México. Terra Latinoamericana, 34, 1 29. Recuperado de http://www.scielo.org.mx/pdf/tl/v34n1/2395-8030-tl-3401-00001.pdf

García-Aranda, M. A., Méndez-González, J., \& HernándezArizmendi, J. Y. (2018). Distribución potencial de Pinus cembroides, Pinus nelsonii y Pinus culminicola en el Noreste de México. Ecosistemas y Recursos Agropecuarios, 5(13), 3-13. doi: 10.19136/era.a5n13.1396

Garza-López, M., Ortega-Rodríguez, J. M., Zamudio-Sánchez, F. J., López-Toledo, J. F., Domínguez-Álvarez, F. A., \& SáenzRomero, C. (2016). Calakmul como refugio de Swietenia macrophylla King ante el cambio climático. Botanical Sciences, 94(1), 43-50. doi: 10.17129/botsci.500

Gutiérrez, E., \& Trejo, I. (2014). Efecto del cambio climático en la distribución potencial de cinco especies arbóreas de bosque templado de México. Revista Mexicana de Biodiversidad, 85(1), 179-188. doi: https://doi.org/10.7550/rmb.37737

Ibarra, M. J., \& Huerta, M. F. (2016). Cambio climático y predicción de incendios al 2050 en el Bosque La Primavera, Jalisco. Revista Mexicana de Ciencias Forestales, 7(37), 39-50. doi: 10.29298/rmcf.v7i37.50.

Instituto Nacional de Estadística y Geografía [Inegi] (2001). Conjunto de datos vectoriales Fisiográficos. Continuo
Nacional escala 1:1000000 serie 1. Subprovincias fisiográficas. Archivo vectorial Shapefile. México, D.F.

Instituto Nacional de Estadística y Geografía [Inegi] (2013). Uso del suelo y vegetación. Escala 1; 250000, serie V (capa unión). Archivo vectorial Shapefile. México, D.F.

Instituto Nacional de Estadística y Geografía [Inegi] (2018). Continuo de elevaciones mexicano. Recuperado de http://www.beta.inegi.org.mx/app/geo2/elevacionesmex/ index.jsp

Islas-Báez, A., Pérez-Miranda, R., González-Hernández, A., Romero-Sánchez, M., \& Velasco-Bautista, E. (2015). Riesgo del hábitat de la Mariposa Monarca (Danaus plexippus) ante escenarios de cambio climático. Revista Ximbai, 11(5), 49-59. Recuperado http://www.redalyc.org/pdf/461/46142593003.pdf

Farjon, A., Pérez de la Rosa, J., \& Styles, T. B. (1997). Guía de campo de los pinos de México y América Central. Royal Botanic Gardens, Kew University of Oxford.

Fernández-Eguiarte, A., Zavala-Hidalgo, J., Romero-Centeno, R., Conde-Álvarez, A. C., \& Trejo-Vázquez, R. I. (2015). Actualización de los escenarios de cambio climático para estudios de impacto, vulnerabilidad y adaptación en México y Centroamérica. México, D. F.: Centro de Ciencias de la Atmosfera, Universidad Nacional Autónoma de México, Instituto Nacional de Ecología y Cambio Climático.

Fick, S. E., \& Hijmans, R. J. (2017). Worldclim 2: New 1-km spatial resolution climate surfaces for global land areas. International Journal of Climatology, 37(12), 4302-4315. doi: 10.1002/joc.5086

Jeschke, J. M., \& Strayer, D. L. (2008). Usefulness of bioclimatic models for studying climate change and invasive species. Annals of the New York Academy of Sciences, 1134(1), 1-24. doi: 10.1196/annals.1439.002

Manzanilla Q., U., \& Aguirre C., O. A. (2017). Zonificación Climática actual y escenarios de cambio climático para la Reserva de la Biosfera Selva el Ocote en Chiapas, México. En L. Ruíz-Montoya, G. Álvarez-Gordillo, N. RamírezMarcial., \& B. Cruz-Salazar (Eds.), Vulnerabilidad social y biológica ante el cambio climático en la Reserva de la Biosfera Selva el Ocote (pp. 25-66). San Cristóbal de las Casas, Chiapas, México: Ecosur.

Manzanilla-Quiñones, U., Martínez-Adriano, C. A., \& AguirreCalderón, O. A. (2019). Modelado espacial histórico y actual del oyamel (Abies religiosa [Kuth] Schltdl. \& Cham.) en la Faja Volcánica Transmexicana. Revista Chapingo Serie Ciencias Forestales y del Ambiente, 25(2), 201-217. doi: 10.5154/r.rchscfa.2018.10.0706 
Martínez-Méndez, N., Aguirre-Planter, E., Eguiarte, L. E., \& Jaramillo-Correa, J. P. (2016). Modelado de nicho ecológico de las especies del género Abies (Pinaceae) en México: Algunas implicaciones taxonómicas y para la conservación. Botanical Sciences, 94(1), 5-24. doi: 10.17129/botsci.508

Martínez-Meyer, E. (2005). Climate change and biodiversity: Some consideradions in forecasting shifts in species potential distributions. Biodiversity Informatics, 2, 45-55. doi: 10.17161/bi.v2i0.8

Miranda, F., \& Hernández-X., E. (1963). Los tipos de vegetación de México y su clasificación. Edición conmemorativa. México, D.F.: Ediciones Científicas Universitarias.

Monterroso-Rivas, A., Gómez-Díaz, J, \& Tinoco-Rueda, J. A. (2012). Bosque mesófilo de montaña y escenarios de cambio climático: Una evaluación en Hidalgo, México. Revista Chapingo Serie Ciencias Forestales y del Ambiente, 19(1), 29-43. doi: $10.5154 /$ r.rchscfa.2012.03.029.

Morrone, J. J., \& Escalante, T. (2016). Introducción a la biogeografía (1a ed.). México, D.F.: Universidad Nacional Autónoma de México.

Monterrubio-Rico, T. C., Charre-Medellín, J. F., PachecoFigueroa, C., Arriaga-Weiss, S., Valdez-Leal, J. D., CancinoMurillo, R., Escalona-Segura, G., Bonilla-Ruíz, C., \& RubioRocha, Y. (2016). Distribución potencial histórica y contemporánea de la familia Psittacidae en México. Revista Mexicana de Biodiversidad, 87(3), 1103-1117. doi: 10.1016/j.rmb.2016.06.0004.

Moore, B., \& Allard, G. (Eds.). (2009). Los impactos del cambio climático en la sanidad forestal. Roma, Italia: Organización de las Naciones Unidas para la Agricultura y la Alimentación (FAO).

Narayani, B. (2008). Tool for Partial ROC (Biodiversity Institute, Lawrence, KS). Ver 1.0. Recuperado de http://nicho.conabio.gob.mx/home/proposito-y-guia-delusuario/validacion-del-modelo

Norby, R., Morgan, J., \& Körner, C. (2007). Ecosystem responses to warming and interacting global change factors. En J. G. Canadell, D. E. Pataki., \& L. F. Pitelka (Eds.), Terrestrial Ecosystems in a Changing World (pp. 23-36). Berlín, Alemania: Springer, Berlin, Heidelberg.

Ortego, J., Gugger, P. F., Riordan, E. C., \& Sork, V. L. (2014). Influence of climatic niche suitability and geographical overlap on hybridization patterns among southern Californian oaks. Journal of Biogeography, 41(10), 1-14. doi: 10.1111/jbi.12334.

Osorio-Olvera, L., Vijay, B., Narayani, B., Soberón, J., \& Falconi, M. (2018). Ntbox: From getting biodiversity data to evaluating species distributions models in a friendly GUI environment. $\mathrm{R}$ package version 0.2.5.4. Recuperado de https://github.com/luismurao/ntbox

Panel Intergubernamental de Expertos sobre Cambio Climático [IPCC] (2007). Cambio climático 2007: Informe de sintesis. Contribución de los grupos de trabajo I, II y III al cuarto informe de evaluación del Panel Intergubernamental de Expertos sobre Cambio Climático. Ginebra, Suiza: IPCC.

Panel Intergubernamental de Expertos sobre Cambio Climático [IPCC] (2013). Resumen para responsables de políticas. Contribución del Grupo de trabajo I al Quinto Informe de Evaluación del Grupo Intergubernamental de Expertos sobre Cambio Climático. En T. F. Stocker, D. Qin, G. K. Plattner, M. Tignor, S. K. Allen, J. Boschung, A. Nauels, Y. Xia, V. Bex., \& P. M. Midgley (Eds.), Cambio Climático 2013: Bases físicas. Reino Unido y Nueva York, Estados Unidos: Cambridge University Press.

Panel Intergubernamental de Expertos sobre Cambio Climático (IPCC). (2014). Resumen para responsables de políticas. Contribución del Grupo de trabajo II al Quinto Informe de Evaluación del Panel Intergubernamental de Expertos sobre el Cambio Climático. En C. B. Field, V. R. Barros, D. J. Dokken, K. J. Mach, M. D. Mastrandrea, T. E. Billir, M. Chatterjee, K. L. Ebi, Y. O. Estrada, R. C. Genova, B. Girma, E. S. Kissel, A. N. Levy, S. MacCracken, P. R. Mastrandrea., \& L. L. White (Eds.), Cambio climático 2014. Impactos, adaptación y vulnerabilidad. Ginebra, Suiza: IPCC.

Pearson, R. G., Raxworthy, C. J., Nakamura, M., \& Peterson, A. (2007). Predicting species distributions from small numbers of occurrence records: a test case using cryptic geckos in Madagascar. Journal of Biogeography, 34(1), 102-117. doi: 10.1111/j.1365-2699.2006.01594.x

Peterson, A. T., \& Nakazawa, Y. (2008). Environmental data sets matter in ecological niche modelling: an example with Solenopsis invicta and Solenopsis richteri. Global Ecology and Biogeography, 17(1), 135-144. doi: 10.1111/j.14668238.2007.00347.x

Peterson, A. T., Papes, M., \& Soberón, J. (2008). Rethinking receiver operating characteristic analysis applications in ecological niche modeling. Ecological Modelling, 213(1), 63-72. doi: 10.1016/j.ecolmodel.2007.11.008

Peterson, A. T. (2011). Ecological niche conservatism: a timestructured review of evidence. Journal of Biogeography, 38(5), 817-827. doi: 10.1111/j.1365-2699.2010.02456.x

Peterson, A. T, Soberón J., Pearson, R. G., Anderson, R. P., Martínez-Meyer, E., Nakamura, M., \& Araujo, M. B. (2011). Ecological Niches and Geographic Distributions. Princeton: Princeton University Press. 
Phillips, S. J., Anderson, R. P., \& Schaphire, R. E. (2006). Maximum entropy modeling of species geographic distributions. Ecological Modelling, 190(3-4), 231-259. doi: 10.1016/j.ecolmodel.2005.03.026

Sáenz-Romero, C., Rehfeldt, G. E., Crookston, N. L., Pierre, D., St-Amant, R., Beaulieu, J., \& Richardson, B. (2010). Contemporary and projected Spline Climate surfaces for Mexico and their use in understanding Climate-plant relationships. Climatic Change, 102(3-4), 595-623. doi: 10.1007/s10584-009-9753-5

Sáenz-Romero, C., Rehfeldt, G. E., Duval, P., \& LindingCisneros, R. A. (2012). Abies religiosa hábitat predicition in Climatic change scenarios and implications for monarch butterfly conservation in Mexico. Forest Ecology and Management, 275, 98-106. doi: 10.1016/j.foreco.2012.03.004

Sáenz-Romero, C., Rehfeldt, G. E., Ortega-Rodríguez, J. M., Marín-Togo M. C., \& Madrigal-Sánchez, X. (2015). Pinus leiophylla Suitable Habitat For 1961-1990 and Future Climate. Botanical Sciences, 93(4), 709-718. doi: $10.17129 /$ botsci.86

Sandoval, V., \& Real, P. (2005). Modelación y prognosis estadística y cartográfica del cambio en el uso de la tierra. Bosque, 26(1), 55-63. Recuperado de https:// scielo.conicyt.cl/scielo.php?script=sci_arttext\&pid $=$ S0717-92002005000100006

Soberón, J., \& Peterson, A. T. (2005). Interpretation of models of fundamental ecological niches and species distribution areas. Biodiversity Information, 2, 1-10. doi: 10.17161/bi.v2i0.4
Sosa-Rodríguez, F. S. (2015). Política del cambio climático en México: avances, obstáculos y retos. Revista Internacional de Estadística y Geografia, 6(2), 4-23. Recuperado de https://www.inegi.org.mx/rde/rde_15/doctos/rde_15_art 1.pdf

Thuiller, W., Lavergne, S., Roquet, C., Boulangeat, I., \& Araujo, M. B. (2011). Consequences of climate change on the tree of life in Europe. Nature, 470, 531-534. doi: 10.1038 / nature09705

Villers, L., \& Trejo, I. (1998). Climate change on Mexican forests and natural protected areas. Global Environmental Change, 8(2), 141-157. doi: 10.1016/S0959-3780(98)00012-0

WorldClim (2018). Free climate data for ecological modeling and GIS. Recuperado de http://www.worldclim.org/CMIP5v1

Manuscrito recibido el 13 de junio de 2018

Aceptado el 23 de noviembre de 2018

Publicado el 29 de julio de 2019

Este documento se debe citar como:

Manzanilla-Quiñones, U., Aguirre-Calderón, O. A., Jiménez-Pérez, J., Treviño-Garza, E. J., \& Yerena-Yamallel, J. I. (2019). Distribución actual y futura del bosque subalpino de Pinus hartwegii Lindl en el Eje Neovolcánico Transversal. Madera y Bosques, 25(2), e2521804. doi: $10.21829 /$ myb.2019.2521804

Madera y Bosques por Instituto de Ecología, A.C. se distribuye bajo una Licencia Creative Commons Atribución-NoComercialCompartirlgual 4.0 Internacional. 